\title{
Modified Medial Gastrocnemius Myocutaneous Flap Technique for Knee Joint Coverage after Total Knee Arthroplasty
}

\author{
Dongseok Kim (D), Junhyung Kim (DD, Woonhyeok Jeong (D), Taehee Jo (D), Jaehoon Choi (BD \\ Department of Plastic and Reconstructive Surgery, Dongsan Medical Center, Keimyung University School of Medicine, Daegu, Korea
}

\begin{abstract}
While there are many reasons the medial gastrocnemius flap is often the favored treatment for soft tissue defects around the knee area, this flap has some disadvantages. Reduced volume at the distal part of the flap and a short reach complicate provision of sufficient coverage for soft tissue defects superior to the patella and the lateral knee. In order to overcome these shortcomings, we modified the typical surgical technique by combining a medial gastrocnemius muscle flap and a medial sural artery perforator flap. This approach was applied to four patients who had developed deep infections and skin and soft tissue defects around the knee joint after total knee arthroplasty. The surgeries were successful. Dead space was well-filled and wounds healed without complications in all patients. This modified medial gastrocnemius myocutaneous flap provides a new option for treating challenging skin and soft tissue defects caused by deep infection after total knee arthroplasty.
\end{abstract}

Keywords: Knee; Arthroplasty, replacement, knee; Reconstructive surgical procedures; Surgical flaps

\section{Introduction}

Soft tissue defects after total knee arthroplasty (TKR) are a daunting problem that have a high probability of revision. The medial gastrocnemius flap is often used to cover such soft tissue defects of the knee or upper third of the leg [1]. The gastrocnemius flap was originally a muscle flap, but in 1978, McCraw et al. [2] demonstrated the possibility of a gastrocnemius myocutaneous flap (MCF). However, there are still few articles reporting knee coverage using a gastrocnemius MCF. Disadvantages of the medial gastrocnemius muscle flap are the reduced volume at the distal part of the flap and the short reach [3]. In order to overcome the limitations of the medial gastrocnemius muscle flap, we combined a medial sural artery perforator flap with a medial gastrocnemius muscle flap, in four cases that required reconstruction of skin and soft tissue defects due to deep infection after TKR. The study was approved by the Institutional Review Board of Keimyung University School of Medicine (IRB No. 2021-02029) and performed in accordance with the principles of the Declaration of Helsinki. Written informed consent was obtained from the patients for the use of their images.

\section{Case}

An 82-year-old woman visited our hospital and was admitted to the orthopedic surgery department because of a deep infection after TKR. Her underlying diseases were

\section{Case Report}

Received: November 12, 2020

Revised: December 11, 2020

Accepted: December 13, 2020

\section{Corresponding author:}

Jaehoon Choi, M.D., Ph.D.

Department of Plastic and Reconstructive Surgery, Keimyung University School of Medicine, 1035 Dalgubeol-daero, Dalseo-gu, Daegu 42601, Korea

Tel: +82-53-258-7815

Fax: +82-53-258-4590

E-mail: diligent78@hanmail.net

This is an Open Access article distributed under the terms of the Creative Commons Attribution Non-Commercial License (https://creativecommons.org/licenses/by-nc/4.0/) which permits unrestricted non-commercial use, distribution, and reproduction in any medium, provided the original work is properly cited.

(c) 2021 Korean Wound Management Society 
hypertension and dementia. Incision and drainage were performed, followed by serial infection control surgery by the orthopedic surgery department. Finally, the infection was controlled, but a skin and soft tissue defect resulted around the

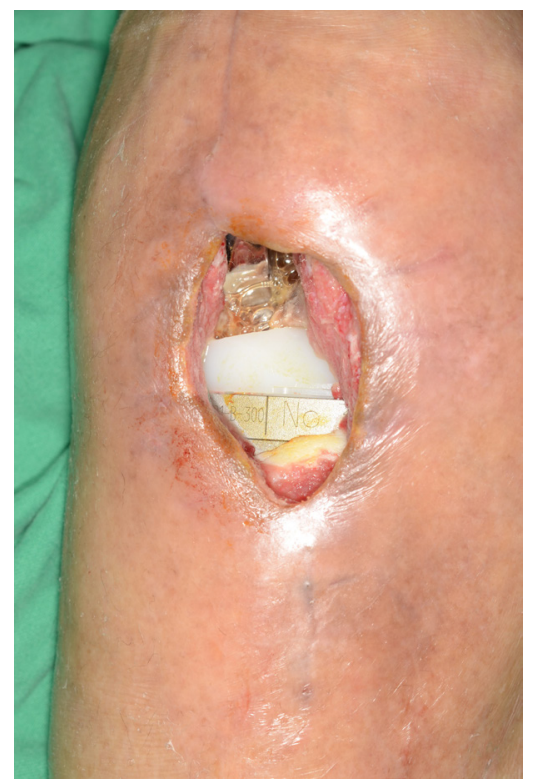

Fig. 1. Preoperative clinical photograph. A $5 \times 3-\mathrm{cm}$ skin and soft tissue defect is seen on the anterolateral area of the knee. The joint capsule is open and the prosthesis is visible.
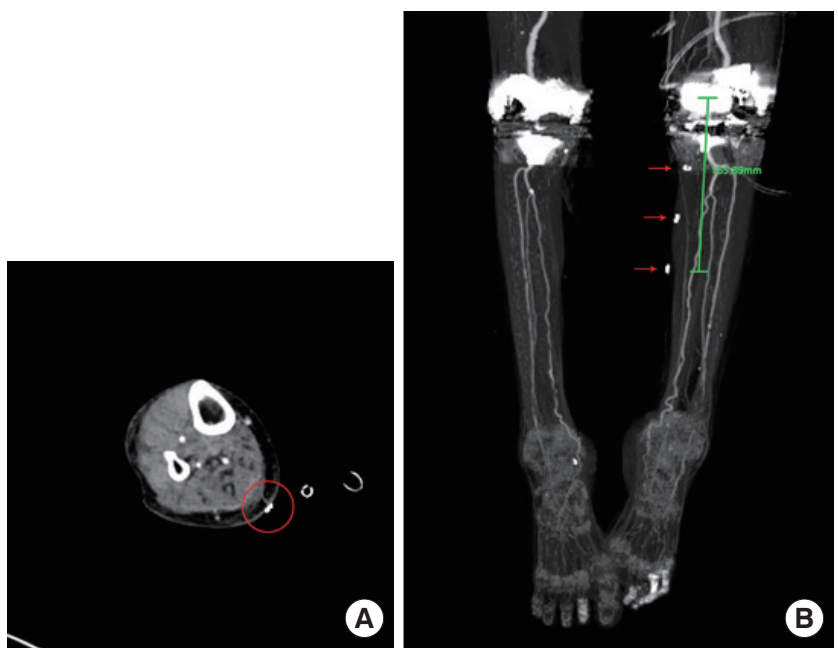

Fig. 2. Preoperative computed tomography angiography of the lower extremities. Before scanning, we used Doppler ultrasonography to find the medial sural artery perforators and marked the location with a skin stapler for visibility on computed tomography. (A) The medial sural artery perforator is observed just beneath the marking (red circle); left and right sides are reversed in the image. (B) Among three perforators (red arrows), we used the most distal perforator which is located about $165 \mathrm{~mm}$ below the popliteal crease; here the image is not reversed. knee. Our department was consulted to cover the defect. The skin defect was located anterolateral to the knee joint (Fig. 1). Since the defect was far from the pedicle of the medial gastrocnemius muscle flap, modifications to the flap were required. Our plan was to elevate the skin flap distal to the gastrocnemius muscle by using a perforator from the most distal part of this muscle. Preoperatively, we used Doppler ultrasonography to find the medial sural artery perforators and marked the location with a skin stapler for visibility on computed tomography (CT). CT angiography of the lower extremities showed the presence of the appropriate perforator near our marking, and we designed the incision line based on the location of the ideal perforator which was located most distally (Figs. 2, 3). Intraoperatively, a preliminary explorative incision was made on the skin and the subfascial plane was carefully dissected from anterosuperior to posteroinferior to locate the perforator. The viability of the perforator was assessed with Doppler ultrasonography (Fig. 4). The flap was elevated and then tunneled and inset to cover the defect (Fig. 5). Flap surgery was successful, and the patient was discharged on foot 2 weeks af-

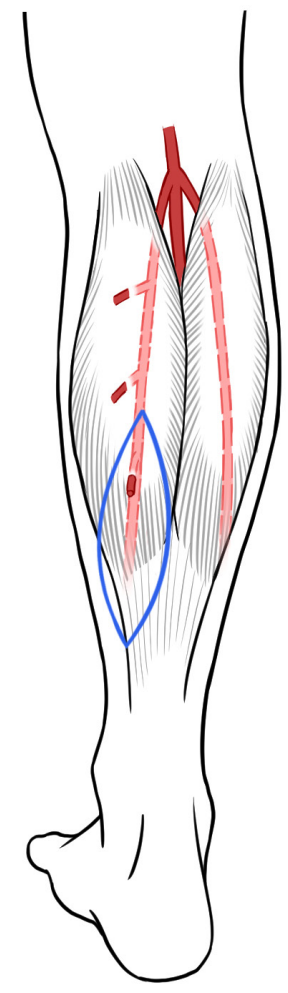

Fig. 3. Design of the modified medial gastrocnemius myocutaneous flap. The marked skin flap (blue line) is elevated together with the medial gastrocnemius muscle flap using the perforator from the most distal part of the gastrocnemius muscle. The left side of the picture is medial, and the right side is lateral. 
Kim D et al.

Infection after total knee arthroplasty

ter surgery (Fig. 6).

\section{Discussion}

Defects on knees are difficult to cover. Among the reconstructive options, local flaps are quite limited because of their small size; other muscular flaps, such as the reverse biceps femoris

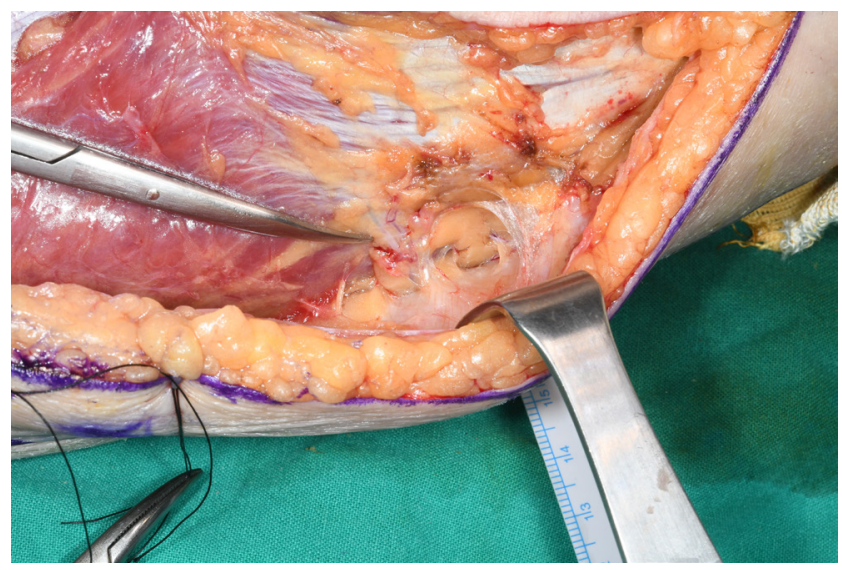

Fig. 4. Intraoperative clinical photograph of case 2 (64-year-old woman). The perforator came from the distal part of the medial gastrocnemius muscle. The viability of the perforator was assessed with Doppler ultrasonography. The left side of the picture is proximal, and the right side is distal. flap, are not superior to the gastrocnemius flap, and have higher donor morbidity. Free tissue transfers can be a good option to cover large and complex wounds, but require significant microsurgical skill and a longer operative time. The medial gastrocnemius muscle flap is, therefore, currently the work-

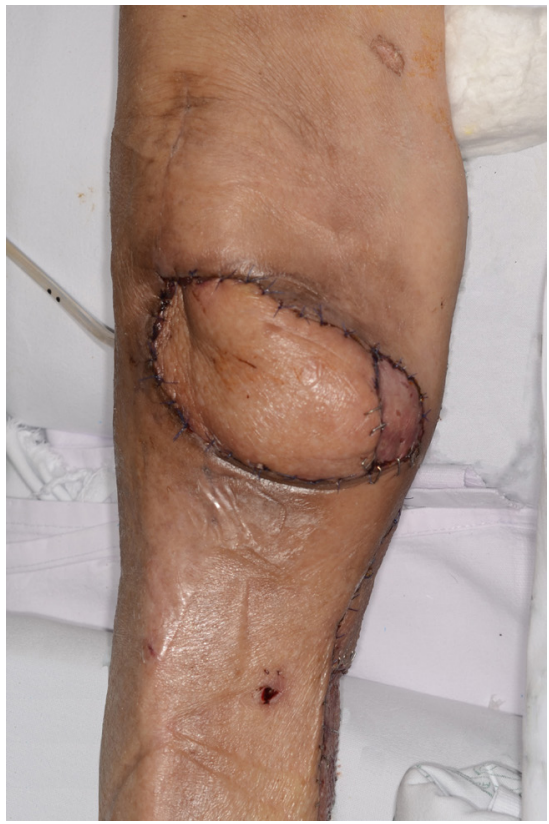

Fig. 6. Postoperative photograph at 1 week after coverage.
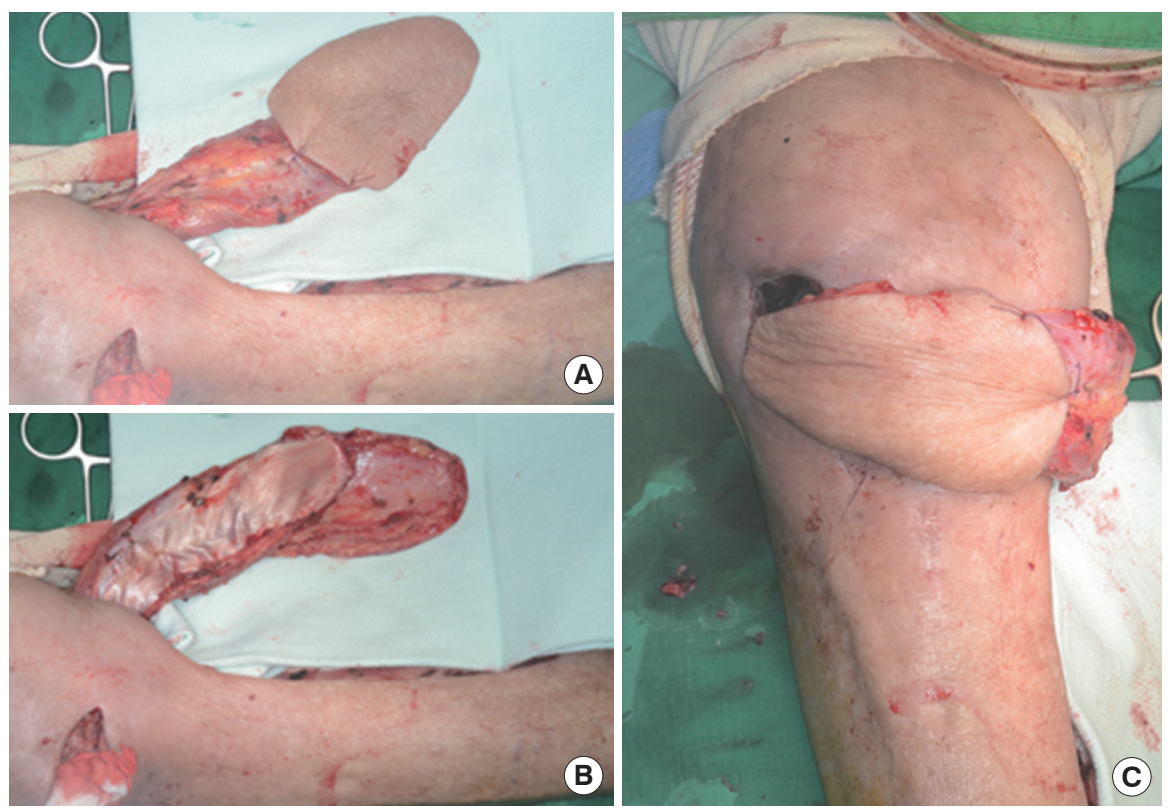

Fig. 5. Intraoperative photograph after the flap was elevated. (A) Upper side of the modified medial gastrocnemius myocutaneous flap. (B) Lower side of the modified medial gastrocnemius myocutaneous flap. The skin flap is elevated more distally than the muscle flap. Without perforator identification, this can lead to total necrosis of the skin paddle at worst. (C) View of the defect with the skin and muscle flap in place. 
horse for covering defects in the anterior aspect of the knee, with its high success rate, ease of harvesting, minimal donorsite morbidity, capacity to fill dead space, and efficiency against infections [4].

However, the disadvantages of the medial gastrocnemius muscle flap include its limited length and the inadequate distal volume of the flap, which make it difficult to cover superior or lateral knee defects. To overcome these disadvantages, surgeons have scored the muscle fascia and performed deep dissections that reach the origin of the muscle [3]. The modified medial gastrocnemius MCF has additional volume on the distal part of the muscle and can reach further than the typical medial gastrocnemius muscle flap. It is effective for wounds that have a gap between the skin defect and the soft tissue defect, and can be used in combination with scoring the fascia or the technique using a deep dissection reaching the pedicle, to

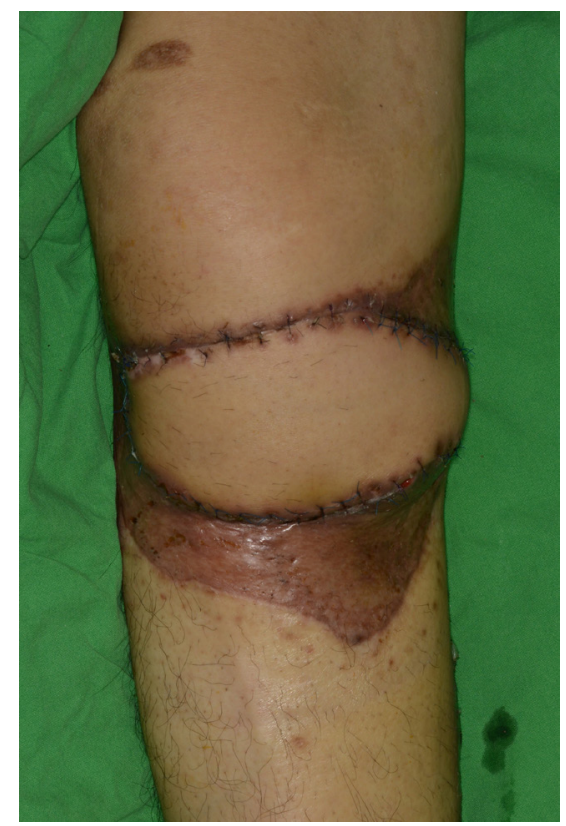

Fig. 7. Postoperative photograph at 2 weeks after coverage (case 4). achieve additional coverage if necessary. It also reduces the need for skin graft on the flap.

The modified medial gastrocnemius MCF was used to treat four serial cases of skin and soft tissue defect after TKR. Two cases had skin defects anteroinferior to the knee joint, one defect was anteromedial, and one was anterolateral. The joint capsule was open in two patients. The size and location of the skin paddle were adapted to each patient's defect, and splitthickness skin grafting was performed as necessary to cover any remnant exposed muscle of the flap or the donor site. All of the flap surgeries aptly covered the defects, and the wounds healed without any major complications that required additional surgery (Table 1, Fig. 7).

According to orthopedic literature, deep infection after TKR occurs in $1 \%$ of cases, and the problem is greatly exacerbated if infection involves the joint capsule [5]. If the joint capsule is open and infection is suspected, the rule is to either change or remove the prosthesis. However, in one of our cases, it was impossible to promptly remove the prosthesis because of instability of the bone. Therefore, the defect was covered just after the infection was controlled and the prosthesis was replaced later by the orthopedic surgery department.

The main difference between our technique and the gastrocnemius MCF technique described by McCraw et al. [2] is the identification of a perforator. This difference allows surgeons to design the skin paddle in the optimum shape to match the defect, without having to lift unnecessarily large skin paddles. Without perforator identification, lifting the skin paddle distal to the gastrocnemius muscle or lifting the arbitrarily-designed skin paddle is less reliable and can lead to total necrosis of the skin paddle at worst.

The perforator from the medial sural artery supplies an average skin area of $13 \times 8 \mathrm{~cm}$, and enables elevation of a larger skin paddle [6]. The selection of the most distal perforator is key to covering further defects, since most people have two or more perforators [6]. However, the shortcoming of this modi-

Table 1. Patient characteristics

\begin{tabular}{|c|c|c|c|c|c|c|}
\hline Case & Age (yr)/sex & Knee skin defect location & Size (cm) & Donor site closure & Comorbidity & Complications \\
\hline 1 & $82 / F$ & Right anterolateral & $5 \times 3$ & Primary closure + STSG & Hypertension, dementia & None \\
\hline 2 & $64 / F$ & Left anteroinferior & $2 \times 2$ & Primary closure + STSG & None & None \\
\hline $3^{\text {a) }}$ & $73 / F$ & Right anteromedial & $3 \times 3$ & Primary closure + STSG & Hypertension, diabetes mellitus & Wound dehiscence \\
\hline 4 & 79/M & Right anteroinferior & $6 \times 8$ & Primary closure & Hypertension & None \\
\hline
\end{tabular}

F, female; M, male; STSG, split-thickness skin graft.

${ }^{a}$ Mild wound dehiscence in case 3 healed well with conservative management. 
fied flap is that it is impossible to perform in patients who do not have a distal perforator. Therefore, precise locating of perforators using CT angiography and Doppler ultrasonography is necessary before surgery.

This modified medial gastrocnemius MCF technique is a simple, new method for improved coverage of large, complex skin and soft tissue defects around the knee. Further research should be conducted to demonstrate clinical safety with a larger number of cases.

\section{Conflict of interest}

No potential conflict of interest relevant to this article was reported.

\section{ORCID iDs}

Dongseok Kim

Junhyung Kim

Woonhyeok Jeong

Taehee Jo

Jaehoon Choi https://orcid.org/0000-0001-9367-6445

https://orcid.org/0000-0001-9077-2853

https://orcid.org/0000-0002-0221-3347

https://orcid.org/0000-0001-8671-6429

https://orcid.org/0000-0001-6180-1619

\section{References}

1. Hallock GG. Anatomic basis of the gastrocnemius perforator-based flap. Ann Plast Surg 2001;47:517-22.

2. McCraw JB, Fishman JH, Sharzer LA. The versatile gastrocnemius myocutaneous flap. Plast Reconstr Surg 1978;62:1523.

3. Cho YJ, Lee JH, Chung DW. Pedicled chimeric gastrocnemius-medial sural artery adipofascial flap for reconstruction of anterolateral defects of the knee. Microsurgery 2017;37: 206-11.

4. Mayoly A, Mattei JC, Moullot P, et al. Gastrocnemius myocutaneous flaps for knee joint coverage. Ann Plast Surg 2018; 81:208-14.

5. Chesney D, Sales J, Elton R, et al. Infection after knee arthroplasty a prospective study of 1509 cases. J Arthroplasty 2008; 23:355-9.

6. Thione A, Valdatta L, Buoro M, et al. The medial sural artery perforators: anatomic basis for a surgical plan. Ann Plast Surg 2004;53:250-5. 